\title{
PALINOLOGÍA Y PALINOFACIES DE UNA SECUENCIA MARINA DEL HOLOCENO MEDIO-TARDÍO: ALBUFERA LANUSHUAIA, CANAL BEAGLE, TIERRA DEL FUEGO, ARGENTINA
}

\author{
MARÍA SOLEDAD CANDEL, MARCELO ADRIÁN MARTÍNEZ \& ANA MARIABORROMEI \\ Instituto Geológico del Sur, CONICET, Departamento de Geología, Universidad Nacional del Sur, San Juan 670, B8000ICN \\ Bahía Blanca, Argentina.scandel@uns.edu.ar,martinez@criba.edu.ar,borromei@criba.edu.ar
}

\begin{abstract}
PALYNOLOGY AND PALYNOFACIES OF A MIDDLE-LATE HOLOCENE MARINE SEQUENCE: ALBUFERA LANUSHUAIA, BEAGLE CHANNEL, TIERRA DEL FUEGO, ARGENTINA. Palynological analysis, with emphasis on palynofacial analysis was carried out on Holocene marine deposits located on the northern coast of the Beagle Channel, Argentina. This analysis allowed paleoenvironmental assessments and paleoclimatic inferences. A fossil section from Albufera Lanushuaia (5452’04.4"S-6760’44.9"O), located on the northern coast of the Beagle Channel was analyzed. The set of particulate organic matter indicate the proximity of the area of continental input to the marine environments, with distances and/or times of transport relatively short. The presence of pyrite suggests reducing conditions, probably associated with marginal-marine environments. At the Albufera Lanushuaia, from the $c a .5,800$ years ${ }^{14} \mathrm{C} \mathrm{BP}$, palynological associations reveal the same pattern of regional vegetation with a closed forest of Nothofagus and an assemblage of Peridiniales dinocysts dominated by taxa that suggest marginal-marine environments, with low to moderate salinity and high nutrient concentrations in shallow marine waters, probably due to the contribution of freshwater due to surface runoff. For the past 3,000 years, a decrease in pollen records of Nothofagus and an increase in shrub and herb pollen with a limited occurrence of aquatic palynomorphs were observed. These reflects the development of a more open forest and adjacent areas of herbaceous and shrub with marginal-marine influence, associated to a regressive event.
\end{abstract}

Key words: palynology, palynofacies, paleoenvironments, middle-late Holocene, Beagle Channel, Argentina.

RESUMO - Análises palinológicas, com ênfase em palinofácies, foram realizadas em depósitos marinhos holocênicos do sul da Argentina, permitindo a obtenção de resultados paleoambientais e paleoclimáticos. O presente estudo baseia-se numa

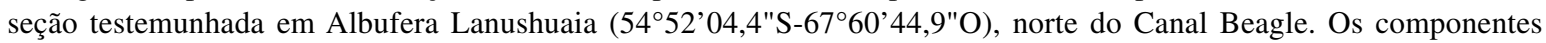
orgânicos particulados recuperados indicam ambiente de deposição marinho com curta distância da área continental e transporte relativamente rápido. A presença de pirita sugere condições redutoras, provavelmente associadas a ambientes marinos marginais. As associações palinológicas revelam que a partir de $5.800{ }^{14} \mathrm{C}$ anos AP a região comportava o mesmo padrão da vegetação regional atual, com uma mata fechada de Nothofagus. Assembleias de dinocistos Peridiniales, dominadas por táxons característicos de ambientes marginais, sugerem condições marinhas rasas, com salinidade de baixa a moderada, altas concentrações de nutrientes, e influência da descarga superficial de águas doces. Para os últimos 3.000 anos, é verificada uma diminuição nos registros de grãos de pólen de Nothofagus e aumento na participação de representantes de ervas e arbustos, além da frequência mais baixa de esporomorfos aquáticos. Esses dados refletem o desenvolvimento de uma floresta mais aberta e áreas adjacentes comportando táxons herbáceos e arbustivos, com influência marinha marginal associada a um evento regressivo.

Palavras-chave: palinologia, palinofácies, paleoambientes, Holoceno médio-superior, Canal Beagle, Argentina.

\section{INTRODUCCIÓN}

El Canal Beagle conecta el extremo sur de los océanos

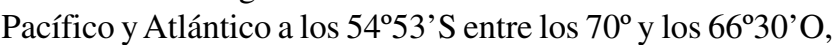
respectivamente. La costa norte corresponde a la Isla Grande de Tierra del Fuego, mientras que la costa sur comprende las islas Hoste y Navarino (Chile), a su vez separadas por el Canal Murray. Es un valle de origen glacial, antiguamente ocupado por un gran glaciar de desagüe proveniente de la Cordillera Darwin, el "Glaciar Beagle". Este valle fue repetidamente glaciado, al menos en dos episodios mayores, durante la "Glaciación Lennox" (Estadío Oxígeno Isotópico 6) y durante la Última Glaciación denominada "Glaciación Moat" (Estadío Oxígeno Isotópico 2) (Rabassa et al., 2000).

El pasaje de lago proglacial a fiordo habría tenido lugar hacia los 8.200 años ${ }^{14} \mathrm{C}$ AP mediante el ingreso de agua de mar por el Canal Murray, quedando el ambiente marino completamente establecido al menos a los 7.900 años ${ }^{14} \mathrm{C} \mathrm{AP}$ (Rabassa et al., 1986). La incursión marina holocena está representada por varias terrazas elevadas discontinuas, 
labradas sobre sedimentos cuaternarios no consolidados o directamente sobre el basamento rocoso, a lo largo de la costa norte del Canal Beagle (Gordillo, 1993). El conjunto de terrazas marinas se subdivide en: Terraza Baja Antigua, Terraza Alta, Terraza Media y Terraza Baja Reciente, depositadas a los 8.000, 6.000, 5.000-3.000 y después de los 3.000 años ${ }^{14} \mathrm{C} \mathrm{AP,}$ respectivamente. Estos depósitos están compuestos básicamente por arenas gruesas que alternan con estratos de rodados. Sedimentos finos, de tipo arcilloso, sólo se encontraron en pequeñas áreas dentro del sector occidental del Canal Beagle (Gordillo, 1993). El control depositacional de este sistema marino es atribuido en parte a factores glacioeustáticos y en parte a movimientos neotectónicos (Rabassa et al., 1992; Bujalesky et al., 2004). Probablemente, las fluctuaciones glacioeustáticas y los movimientos corticales glacioisostáticos habrían actuado hasta los 5.000 años ${ }^{14} \mathrm{C}$ AP, momento en el cual el nivel eustático del mar se estabiliza. La actividad neotectónica sería posterior a los 5.000 años AP continuando hasta la actualidad (Gordillo, 1993).

Existen estudios geológicos, geomorfológicos y paleoecológicos realizados en Tierra del Fuego sobre los cambios del nivel del mar durante el Holoceno, pudiéndose mencionar entre otros: Codignotto (1984), Porter et al. (1984), Rabassa et al. (1986, 1992, 2000), Isla (1989), Rutter et al. (1989) y Gordillo et al. (1992, 1993). Hasta el momento, los únicos estudios sobre paleomicroplancton marino de pared orgánica y palinofacies han sido realizados por Borromei et al.(1997), Borromei \& Quattrocchio (2001, 2007), Grill et al. (2002), Candel et al. (2009) y Rabassa et al. (2009), sobre depósitos marinos holocenos aflorantes a lo largo del Canal Beagle.

El objetivo de este trabajo es contribuir al conocimiento de las condiciones ambientales y climáticas existentes durante la depositación de los sedimentos marinos holocenos en el sur de Tierra del Fuego, en base al análisis palinológico y palinofacies (estudio del contenido orgánico palinológico total). La comparación de los sedimentos marinos de Albufera Lanushuaia con aquellos de las localidades de Bahía Lapataia, Río Varela y Río Ovando permitirá interpretar las condiciones paleoambientales existentes durante los cambios del nivel del mar holoceno en el área del Canal Beagle.

\section{CARACTERÍSTICAS DEL ÁREA DE ESTUDIO}

\section{Ubicación geográfica del Canal Beagle}

El Canal Beagle se comunica, en su extremo oeste, con el Océano Pacífico mediante los brazos Noroeste y Sudoeste rodeando la isla Gordon. Desde la punta que divide estos brazos (Punta Divide) hacia el este, el canal tiene una extensión de $180 \mathrm{~km}$. La costa norte corresponde a la Isla Grande de Tierra del Fuego, mientras que la costa sur comprende las islas Hoste y Navarino, a su vez separadas por el Canal Murray. Las islas Lennox y Nueva, aflorantes desde una plataforma de $50 \mathrm{~m}$ de profundidad, quedan incluidas en el ámbito del canal (Figura 1). Las profundidades máximas alcanzan valores entre 240 y 300 m al oeste de la conexión con el Canal Murray, mientras que hacia el este las profundidades son menores. El ancho medio es de $5 \mathrm{~km}$, alcanzando valores mínimos en el Archipiélago Gable con 1,8 $\mathrm{km}$ (Paso Mackinlay). La isla Gable a su vez constituye un umbral de solo $30 \mathrm{~m}$ de profundidad (Banco Gable) (Isla et al., 1999).

En este valle glaciario se reconoce la presencia de escalones de artesa o umbrales, arcos morénicos frontales, morenas basales, campos de drumlins y fondos lacustres. Entre las geoformas aflorantes se destacan el conjunto de rocas aborregadas de las islas Bridges y otros islotes ubicados en la boca de la Bahía Ushuaia, y el campo de drumlins que conforma el Archipiélago Gable (Rabassa in Isla et al., 1999).

Las aguas del Canal Beagle están influenciadas por una fuerte descarga de agua dulce de las precipitaciones y glaciares a través de los ríos durante el verano. La columna de agua presenta una fuerte estratificación termohalina con una mezcla de agua a los $12 \mathrm{~m}$ de profundidad principalmente durante la estación de verano. La temperatura promedio del agua superficial del mar es de $6,5^{\circ} \mathrm{C}$ con un máximo de $9^{\circ} \mathrm{C}$ en enero y un mínimo de $4^{\circ} \mathrm{C}$ en agosto. La salinidad del agua superficial del mar varía entre 27 a 33,5 UPS (Unidades Prácticas de Salinidad). Los valores mínimos de salinidad son obtenidos durante el verano, dependiendo del volumen de descarga por derretimiento dentro del canal. El Canal Beagle tiene condiciones libres de hielo a lo largo de todo el año (Iturraspe et al., 1989; Isla et al., 1999). La dinámica estuarina está controlada por las fuentes de agua dulce estacionales y por los flujos de mareas desde el este (Atlántico) y desde el oeste (Pacífico) (Isla et al., 1999).

\section{Albufera Lanushuaia}

Se encuentra ubicada a $5,7 \mathrm{~km}$ al este del casco de la Estancia Harberton y a 2,7 km aproximadamente al oeste de la localidad Río Varela, sobre la costa norte del Canal Beagle (Figura 1). Corresponde a un ambiente actual de albufera o marisma. El paisaje se caracteriza por formas del relieve originadas por acumulación subglacial pertenecientes al campo de drumlins en Estancia Harberton-Isla Gable. Estas formas fueron generadas por el hielo durante la Última Glaciación o "Glaciación Moat” (20.000 años AP) (Rabassa et al., 2000).

La secuencia estudiada (5452'04.4"S-6760’44.9"O) comprende $0,95 \mathrm{~m}$ de profundidad desde la base al techo, observándose:

(i) Unidad litológica $C(0,95-0,34 \mathrm{~m})$. Arcilla gris verdosa $\mathrm{y}$ arena muy fina. Abundante materia orgánica vegetal y restos de bivalvos (Mytilus sp.). Dataciones: $5.750 \pm 80$ y $5.560 \pm$ 110 años ${ }^{14} \mathrm{C}$ AP (Tabla 1);

(ii) Unidad litológica $B(0,34-0,12 \mathrm{~m})$. Arcilla gris verdosa $\mathrm{y}$ arena muy fina. Escasa materia orgánica vegetal, sin valvas;

(iii) Unidad litológica A $(0,12-0 \mathrm{~m})$. Arcilla con gravilla. Fango fétido negro. Abundante materia orgánica vegetal. Datación radiocarbónica: Post-bomb (Tabla 1).

\section{MATERIAL Y MÉTODOS}

Mediante la utilización de un muestreador tipo Ruso (Wardenaar) se extrajo un testigo de 0,95 $\mathrm{m}$ de espesor, el 
cual fue submuestreado cada $1 \mathrm{~cm}$. Para el análisis palinológico se procedió al estudio de 27 muestras, a intervalos de $4 \mathrm{~cm}$. El tratamiento físico-químico de las muestras fósiles se realizó según las técnicas de Heusser \& Stock (1984), con modificaciones de Dale (1976) para la preservación del paleomicroplancton marino de pared orgánica. Las muestras fueron tratadas con ácidos en frío (HCL y HF) y se omitió la acetólisis y oxidación con la finalidad de preservar el microplancton de pared orgánica así como también el contenido orgánico de las palinofacies inalterado. Se agregaron tabletas con esporas exóticas (Lycopodium clavatum) para el cálculo de la concentración palinológica (palinomorfos/gramo de sedimento seco) (Stockmarr, 1971). El residuo fue filtrado por tamiz de $10 \mu \mathrm{m}$ para remover las partículas finas y concentrar el material particulado. Se confeccionaron preparados para el análisis palinológico tradicional y para el análisis palinofacial teniendo en cuenta la técnica de Tyson (1995), la cual consiste en contar 500 partículas de materia orgánica mayores a $10 \mu \mathrm{m}$. El material fue estudiado mediante microscopia de luz transmitida y fluorescencia. Los recuentos palinológicos y análisis palinofacial se llevaron a cabo con un aumento de x400 y la determinación sistemática se realizó sobre la base de las características morfológicas y morfométricas de los esporomorfos y palinomorfos acuáticos con un aumento de $\mathrm{x} 1.000$. Las muestras palinológicas se encuentran archivadas en el Laboratorio de Palinología, Universidad Nacional del Sur, bajo la denominación UNSP seguido por las siglas correspondientes a la sección estudiada: AL (Albufera Lanushuaia).

Los palinomorfos identificados fueron agrupados en: árboles, arbustos y hierbas, acuáticas y criptógamas, algas, dinoquistes marinos, acritarcos, huevos de copépodos, foraminíferos y otros palinomorfos acuáticos. El estudio sistemático de los quistes de dinoflagelados actuales del Canal Beagle fue realizado en el Centre de recherché en géochimie et géodynamique (GEOTOP-UQAM-McGill, Université du Québec à Montréal) Montreal, Canadá, bajo la dirección de Anne de Vernal y Taoufik Radi. Con el objetivo de efectuar evaluaciones paleoambientales, los distintos taxones reconocidos en esta contribución fueron comparados con sus equivalentes actuales (Candel, 2010). Se incluyen en el apéndice taxonómico solamente las descripciones de dinoquistes con asignaciones abiertas tales como cf. Dubridinium sp. y cf. Selenopemphix quanta.

De acuerdo con Heusser (1998), Nothofagus betuloides, $N$. pumilio y $N$. antarctica fueron incluidos dentro del grupo taxonómico $N$. tipo dombeyi dado que los granos de polen poseen una morfología similar dificultando su diferenciación. Lo mismo ocurre con Empetrum rubrum y Gaultherial Pernettya (Ericaceae), cuyos granos de polen son muy similares y ocurren en tétradas, por lo tanto son considerados

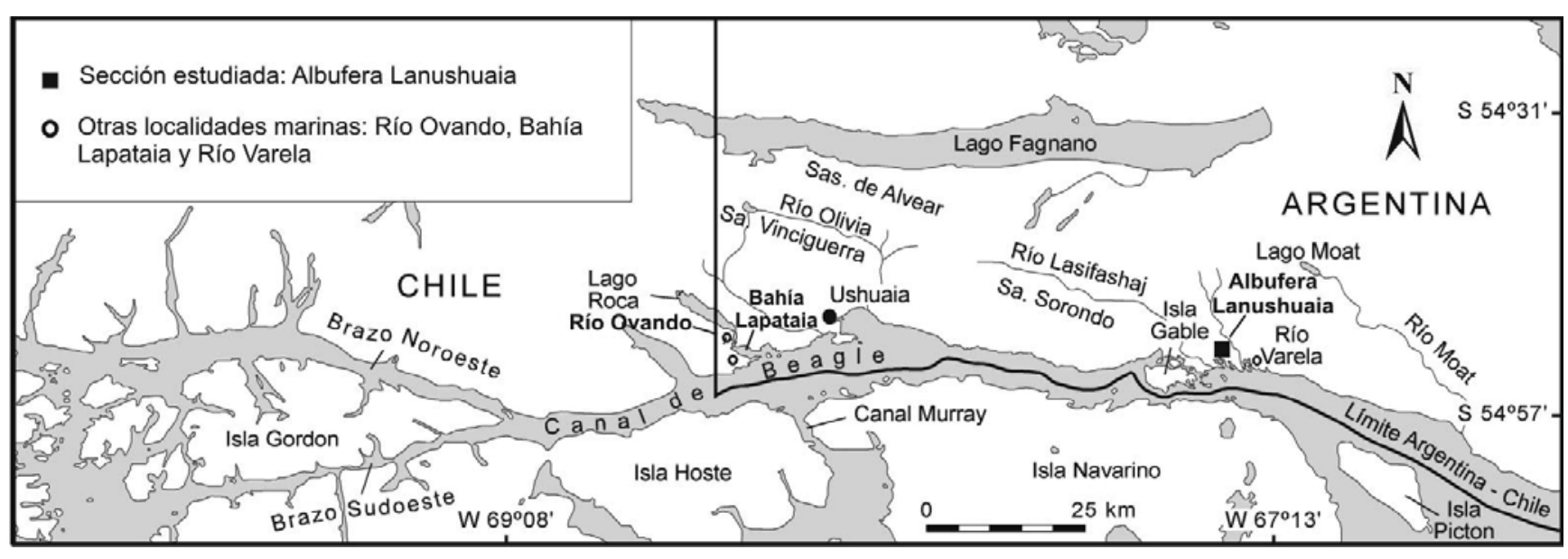

Figura 1. Mapa de ubicación del área de estudio.

Figure 1. Location map of the study area.

Tabla 1. Dataciones radiocarbónicas y edades calibradas de la sección Albufera Lanushuaia.

Table 1. Radiocarbon dating and calibrated ages from the Albufera Lanushuaia section.

\begin{tabular}{ccccccc}
\hline $\begin{array}{c}\text { Profundidad } \\
(\mathrm{cm})\end{array}$ & $\begin{array}{c}\text { Referencia } \\
\text { Laboratorio } \\
\mathrm{N}^{0}\end{array}$ & $\begin{array}{c}\text { Años } \mathrm{C}^{14} \\
\text { A.P. }\end{array}$ & $\begin{array}{c}\text { Años calibrados } \\
\text { A.P. (probabilidad } \\
\text { media) }\end{array}$ & rango $1 \sigma$ & rango 2 $\sigma$ & Material \\
\hline 3 & AA86258 & Post-bomb & 1958 (D.C.) & $\begin{array}{c}1957.91- \\
1958.41\end{array}$ & $\begin{array}{c}1957.85- \\
1958.63\end{array}$ & $\begin{array}{c}\text { Materia } \\
\text { orgánica }\end{array}$ \\
37 & Pta8523 & $5560 \pm 110$ & 6298 & $6184-6413$ & $6426-6435$ & $\begin{array}{c}\text { Materia } \\
\text { orgánica } \\
\text { Materia } \\
\text { orgánica }\end{array}$ \\
\hline
\end{tabular}


como un mismo grupo taxonómico en los diagramas polínicos denominado tipo Empetrum/Ericaceae.

La nomenclatura taxonómica de los quistes de dinoflagelados utilizada en este trabajo corresponde a Rochon et al. (1999), Head et al. (2001) y Zonneveld (1997). Los taxones de quistes de dinoflagelados aquí estudiados han sido citados siguiendo a Fensome \& Williams (2004). La asignación de los taxones de dinoquistes fósiles a nivel específico fue efectuada por comparación con formas modernas halladas en las muestras superficiales del fondo del Canal Beagle (Candel, 2010). Los especímenes de Brigantedinium simplex Wall, 1965 y B. cariacoense Wall 1967 fueron agrupados bajo el nombre de Brigantedinium spp., cuando el arqueopilo no pudo ser observado claramente debido a la orientación de los ejemplares y/o pobre preservación del material. Dentro del grupo "round brown indeterminados" se incluyen informalmente aquellos dinoquistes de cuerpos redondeados, pared lisa y pigmentada de color marrón (Protoperidinoideos). De la misma manera, aquellos dinoquistes proximocorados que no pudieron ser asignados a un género determinado fueron agrupados en la categoría "proximocorados indeterminados". Las algas correspondientes a Zygnemataceae, Spirogyra, tipo 128 (Pals et al., 1980) y 181 (van Geel et al., 1983), son agrupadas en "algas dulceacuícolas" mientras que las algas pertenecientes a Prasinophyceae (Tasmanaceae) se incluyen dentro de "algas marino-salobres". Cabe destacar que si bien estos grupos de algas poseen distintos requerimientos ecológicos, ambos evidencian descarga fluvial al ambiente marino, ya sea como componente alóctono (presencia de algas dulceacuícolas en ambiente marino marginal) o bien como componente autóctono (algas marino-salobres), donde la descarga fluvial genera salinidades subnormales favoreciendo la proliferación de las mismas. Todos aquellos ejemplares de microplancton cuya mala preservación no permitió la inclusión en las categorías previamente mencionadas, fueron incluidos en el grupo "palinomorfos acuáticos indeterminados". En el apéndice taxonómico se incluyen descripciones de ejemplares de microplancton de pared orgánica con asignación abierta y corresponde a Candel (2010).

Los recuentos palinológicos se realizaron sobre un mínimo de 200 palinomorfos. Además, para garantizar la presencia de los taxones menos representados, los preparados fueron recorridos en su totalidad. Los taxones así identificados, aunque quedan fuera del recuento, son incorporados como presencia en el diagrama palinológico con el objetivo de caracterizar en su conjunto a los palinomorfos terrestres y marinos.

La estimación de las frecuencias relativas (\%) de los palinomorfos terrestres (granos de polen y esporas) y de los palinomorfos acuáticos (dinoquistes, acritarcos, huevos de copépodos, foraminíferos, algas) se calculó sobre el total de palinomorfos contados. La concentración polínica o Frecuencia Polínica Absoluta (APF) se define como el número de ejemplares de palinomorfos por unidad de volumen o masa de sedimento seco o húmedo (Birks \& Birks, 1980). En esta contribución se ha trabajado con sedimentos secos expresando la concentración en palinomorfos/gramo.

La zonación del perfil fósil fue realizada mediante "Cluster Analysis" (CA) Constrained single-link (Birks \& Gordon, 1985) utilizando como coeficiente de disimilitud la distancia de Edwards \& Cavalli-Sforza (Overperck et al., 1985; Prentice, 1986).

Para el análisis palinofacial se realizaron recuentos de un mínimo de 500 partículas mayores a $10 \mu \mathrm{m}$. La clasificación de la materia orgánica palinológica utilizada es la propuesta por Tyson (1995) quien divide la misma en cuatro tipos principales: palinomorfos, fitoclastos (translúcidos y opacos), amorfo y zooclastos.

La estimación de las frecuencias relativas (\%) de palinomorfos, fitoclastos translúcidos, fitoclastos opacos, amorfo y zooclastos se calculó sobre el total de las partículas contadas. Si bien, las frecuencias del grupo palinomorfos fueron llevadas a cabo considerando el total de partículas contadas (500 como mínimo), los porcentajes de cada uno de los constituyentes de dicho grupo (granos de polen, esporas y microplancton) se realizó sobre el recuento palinológico (200 palinomorfos como mínimo).

El deterioro que presentan los palinomorfos y la materia orgánica en general se determinó de acuerdo a Delcourt \& Delcourt (1980), quienes reconocen cuatro tipos de deterioro: corrosión (producida por oxidación bioquímica relacionada a actividad local de bacterias y hongos); degradación (oxidación química en ambientes aéreos y subaéreos); daño mecánico (por transporte físico y/o compactación sin y postdeposicional dentro de los sedimentos); y cristalización de pirita (diagénesis con precipitación de minerales autigénicos). Esta herramienta permite hacer inferencias sobre las condiciones paleoambientales.

La representación de las frecuencias relativas y concentración de palinomorfos y de la materia orgánica, los diagramas y el análisis de agrupamiento se realizaron con el programa TGview 2.0.2 (Grimm, 2004).

Las dataciones radiocarbónicas sobre materia orgánica fueron realizadas en el CSIR Environmentek, Pretoria, Sudáfrica y AMS Facility, University of Arizona, Tucson, U.S.A. (Tabla 1). La calibración se realizó con el programa Calib Rev 5.1.0 (Stuiver \& Reimer, 1993; Stuiver et al., 2005), curva de calibración del Hemisferio Sur (SHcal04.14c; McCormac et al., 2004). La calibración de sedimentos con edad radiocarbónica posterior a 1.950 fue efectuada con el programa CALIBomb (Reimer et al., 2004). En el presente trabajo se utilizarán los fechados radiocarbónicos con el fin de comparar con otras localidades marinas en el área del Canal Beagle. Se debe considerar un efecto reservorio de $556 \pm 61$ años, según lo analizado por Albero et al. (1988) para el área del Canal Beagle.

\section{RESULTADOS}

En la secuencia fósil de Albufera Lanushuaia se reconocieron dos zonas palinológicas en base al análisis de 
agrupamiento. La Zona Palinológica AL-1 comprende la parte superior de la Unidad Litológica A y la Zona Palinológica AL2, la parte inferior de la Unidad Litológica A y las unidades litológicas B y C (Figura 2). Basado en los componentes acuáticos, la Zona Palinológica AL-2 fue subdividida en dos subzonas palinológicas: AL-2a y AL-2b. Las palinofacies son descriptas para cada zona y subzona palinológica. Los valores de las frecuencias relativas correspondientes a los cuatro grupos de la materia orgánica palinológica fueron expresados como valores mínimo-(promedio)-máximo. De abajo hacia arriba de la secuencia, se reconocen las zonas palinológicas abajo descriptas.

\section{Zona Palinológica AL-2}

Se reconocieron los cuatro grupos de materia orgánica palinológica. Palinomorfos: 1-(3,3)-6,1\%; fitoclastos: 87-(93,7)97\%; amorfo: 1-(2,6)-6,5\%; y zooclastos: 0,2-(0,4)-1,2\% (Figura2).

Dentro del grupo palinomorfos, esta zona se encuentra dominada por Nothofagus tipo dombeyi cuyas frecuencias relativas varían entre 85,6 y 94,6\%. Acompañan Asteraceae subf. Asteroideae, Empetrum/Ericaceae y Poaceae con frecuencias inferiores a 3,8\%. Gunnera, Apiaceae, Acaena y Lycopodiaceae registran cada uno valores $<2 \%$ (Figura 3).

Los restantes constituyentes de la materia orgánica palinológica se caracterizan por el predominio de fitoclastos translúcidos principalmente no-bioestructurados. Los fitoclastos opacos están escasamente representados. Acompañan materia orgánica amorfa granular en transición a esponjoso y zooclastos.

Subzona AL-2b. Se reconocieron los cuatro grupos de materia orgánica palinológica. Palinomorfos: 1-(3,6)-6,1\%; fitoclastos: 87-(93)-95,9\%; amorfo: 1,4-(2,9)-6,5\%; y zooclastos: 0,2-(0,5)$1,2 \%$ (Figura 2).
El grupo palinomorfos registrado en esta subzona presenta un bajo contenido en sus constituyentes microplanctónicos (Figura 3). La asociación de dinoquistes alcanza hasta $1,6 \%$ y está representada por Brigantedinium spp., Echinidinium spp., Islandinium minutum (Harland \& Reid en Harland et al.) Head et al., cf. Dubridinium sp., Selenopemphix nephroides (Benedek) Benedek \& Sarjeant, quistes proximocorados y "round brown" indeterminados. Otros componentes acuáticos registrados con bajos valores son Halodinium sp. con frecuencias de hasta 1\%, Acritarco sp. 1 alcanzando hasta $1,9 \%$ y foraminíferos con valores $<1 \%$. Los huevos de copépodos presentan los valores más altos, alcanzando hasta 2,3\%. La asociación de algas (Tasmanaceae, Spirogyra sp., Zygnemataceae, tipo 128 y 181 van Geel) está presente con valores entre 0,3 y $1,7 \%$ (Figura 4 ).

Los restantes componentes de la materia orgánica palinológica se caracterizan por el predominio de fitoclastos translúcidos en su mayoría no-bioestructurados de color amarillo pálido a castaño oscuro, pseudoamorfos y en algunos casos con estructura relíctica fibrosa y/o bandeada con bordes subredondeados, difusos o corroídos. Dentro de los bioestructurados, las hifas de hongos son abundantes junto con otros fitoclastos cuyo origen no pudo determinarse claramente. Además acompañan en bajas proporciones, cutículas y traqueidas. La pirita está presente formando masas aglutinadas o diseminada sobre los fitoclastos. Los fitoclastos opacos son muy escasos en forma de tablillas de tamaño pequeño generalmente sin bioestructura y, en menor abundancia, de tipo equidimensionales, angulosos a subredondeados de tamaño pequeño. El material amorfo presente es predominantemente esponjoso, en algunos casos transicional a amorfo granular. Dentro de los zooclastos se registran piezas esqueletales de invertebrados (Figura 5).

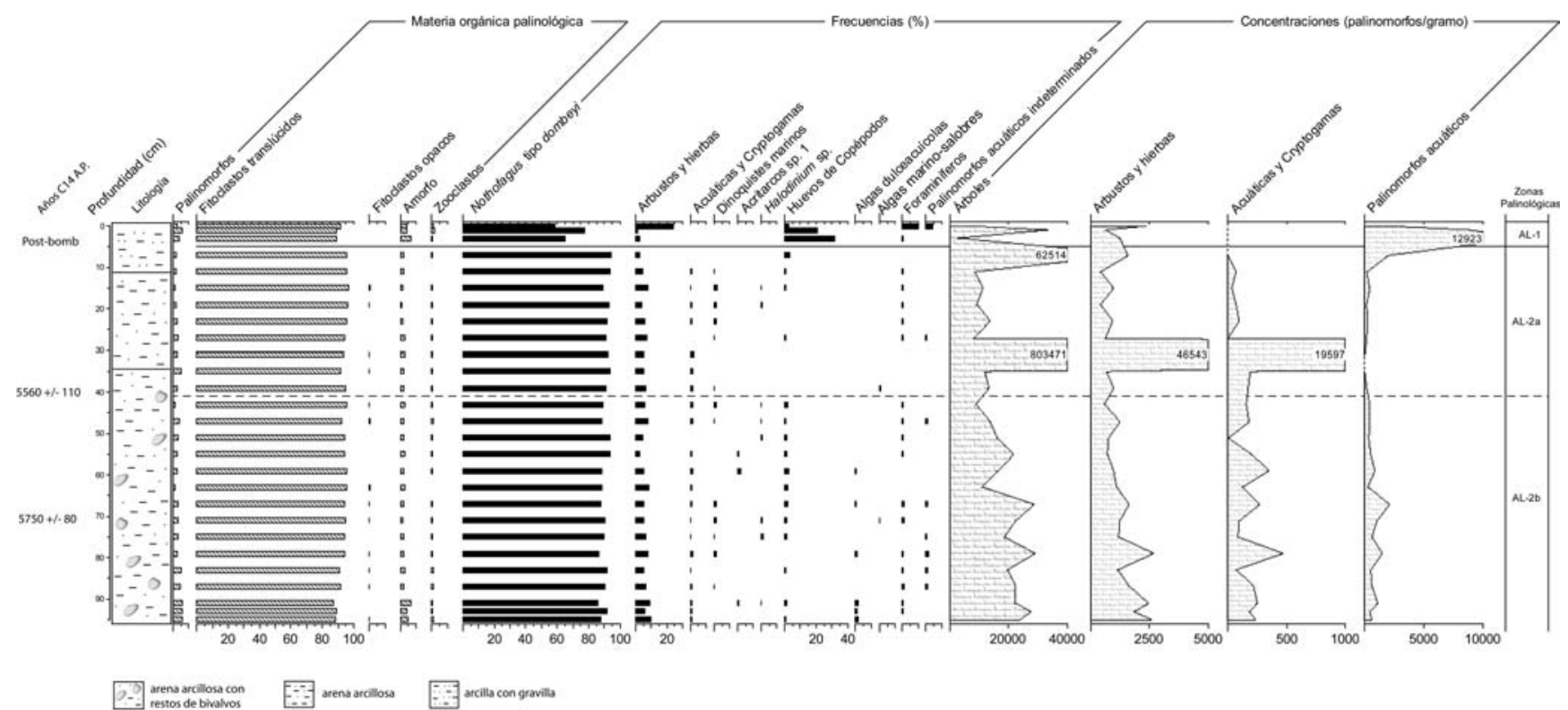

Figura 2. Frecuencias relativas y concentraciones de las asociaciones microflorísticas y palinofacies de la sección Albufera Lanushuaia.

Figure 2. Relative frequencies and concentrations of microfloristic assemblages and palynofacies from the Albufera Lanushuaia section. 
Subzona AL-2a. Las palinofacies se hallan dominadas por fitoclastos: 92-(95,1)-96,6\%, seguidos de palinomorfos: 1,4(2,6)-5,4\%; amorfo: 1-(2)-2,9\%; y zooclastos: 0,2-(0,3)-0,6\% (Figura 2).

Los palinomorfos acuáticos muestran bajas frecuencias relativas (Figura 3). Los dinoquistes alcanzan valores de hasta $2 \%$ y están representados por Brigantedinium spp., Echinidinium spp., Selenopemphix quanta (Bradford) Matsuoka, cf. Selenopemphix quanta, Votadinium spinosum Reid, Polykrikos kofoidii Chatton, P. schwartzii Bütschli, quistes proximocorados y "round brown" indeterminados. Halodinium sp. y foraminíferos también registran valores inferiores a $1 \%$, mientras que los huevos de copépodos alcanzan hasta $3 \%$ (Figura 4).

Los restantes constituyentes de la materia orgánica palinológica presentan alta abundancia de fitoclastos translúcidos fundamentalmente con formas nobioestructuradas, de color amarillo pálido hasta castaño oscuro, pseudoamorfos o gelificados, altamente degradados, en menor proporción fibrosos y/o bandeados. En cuanto a los fitoclastos bioestructurados, están presentes hifas de hongos, cutículas y otros bioestructurados. Los fitoclastos opacos son escasos con formas de tablillas sin bioestructura y tamaño pequeño. El amorfo es de tipo esponjoso, en algunos casos con transición a granular. Los zooclastos están representados por piezas esqueletales de invertebrados.

Concentración polínica y palinomorfos acuáticos. El polen arbóreo en la zona palinológica Al-2 registra valores que varían entre 7.991 y 803.471 granos/gramo y el polen de arbustos y hierbas entre 397 y 46.542 granos/gramo. La asociación de palinomorfos acuáticos muestra valores de concentración que varían entre 86 y 2.021 ejemplares/gramo. Los valores más altos (2.021 y 1.959 ejemplares/gramo) se registran a los 67 y $7 \mathrm{~cm}$, principalmente por huevos de copépodos.

\section{Zona Palinológica AL-1}

Se reconocieron los cuatro grupos de materia orgánica palinológica. Palinomorfos: 2,8-(4)-5,5\%; fitoclastos: 88,8-(90)91,8\%; amorfo: 3,9-(5)-6,9\%; y zooclastos: 0,4-(1)-1,6\% (Figura 2).

Dentro de los palinomorfos, se observa un incremento de Poaceae (18\%), Caryophyllaceae (4,3\%) y Gunnera $(1,4 \%)$. Acompañan Asteraceae subf. Asteroideae, Empetrum/ Ericaceae con valores inferiores a $1 \%$. Las frecuencias de Nothofagus tipo dombeyi muestran una disminución respecto de la zona anterior (Zona AL-2) con valores entre 58,6 y 77,6\%. Los palinomorfos acuáticos están representados por huevos de copépodos, foraminíferos y palinomorfos acuáticos indeterminados con valores de hasta $31,7 \%$ (Figura 3 ).

Respecto de los demás constituyentes de la materia orgánica palinológica, se observa un predominio de fitoclastos translúcidos, principalmente no-bioestructurados pseudoamorfos y gelificados, altamente degradados, asociados con otros de estructura relíctica fibrosa y/o bandeada, de color amarillo pálido a castaño. Los fitoclastos bioestructurados están representados principalmente por hifas de hongos y cutículas. Los fitoclastos opacos están ausentes en esta zona. La materia orgánica amorfa es esponjosa mostrando en algunos casos transición a granular. La presencia de pirita se observa en forma diseminada y sobre los fitoclastos translúcidos (Figura 5).

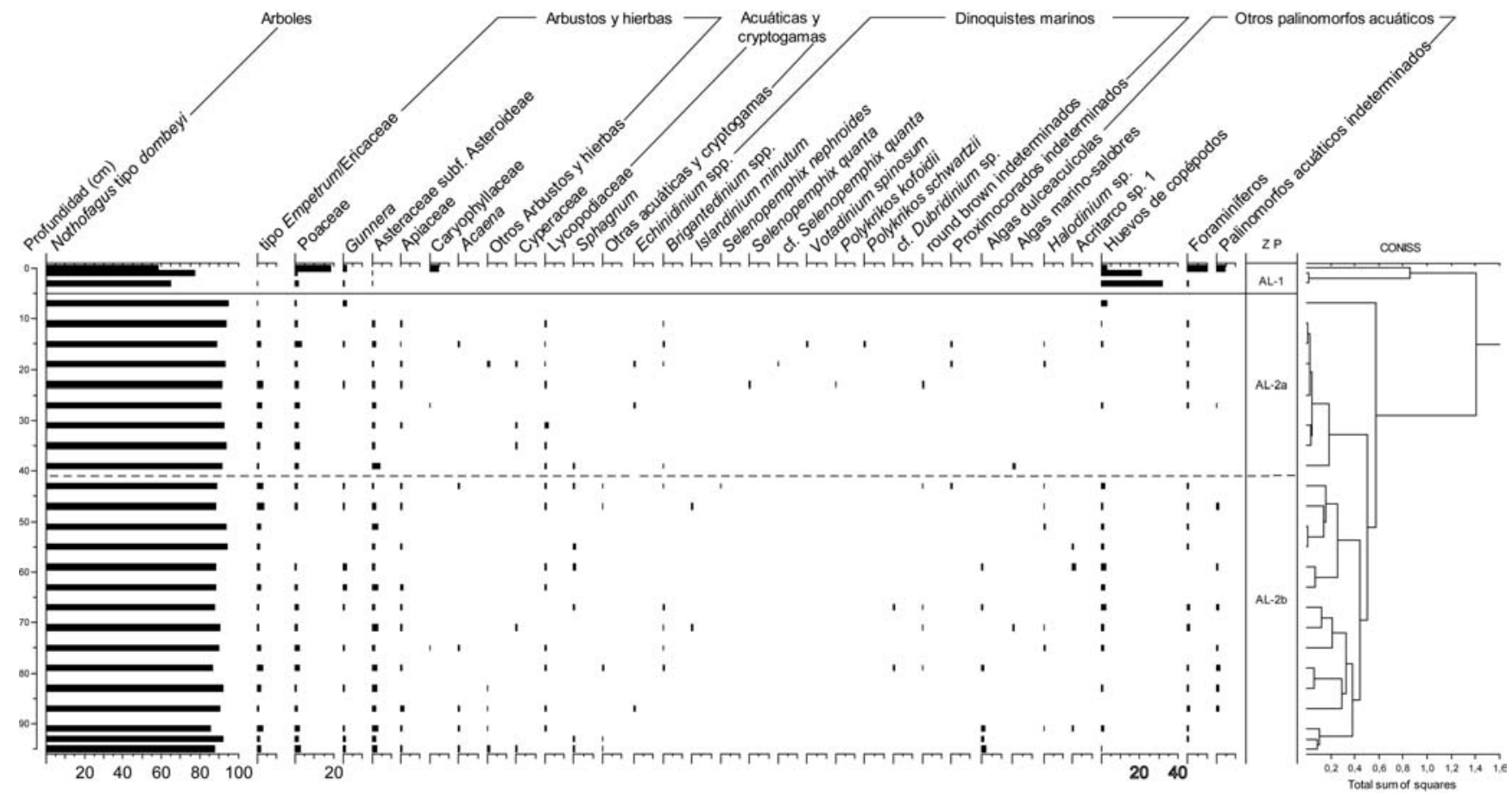

Figura 3. Frecuencias relativas de los componentes polínicos y acuáticos de la sección Albufera Lanushuaia.

Figure 3. Relative frequencies of pollen and aquatic components from the Albufera Lanushuaia section. 
Concentración polínica y de palinomorfos acuáticos. Los valores de concentración del polen arbóreo en esta zona (Al1) varían entre 2.639 y 33.283 granos/gramo y la concentración de arbustos y hierbas entre 614 y 2.340 granos/gramo. Las concentraciones de los palinomorfos acuáticos varían entre 1.652 y 12.922 ejemplares/gramo.
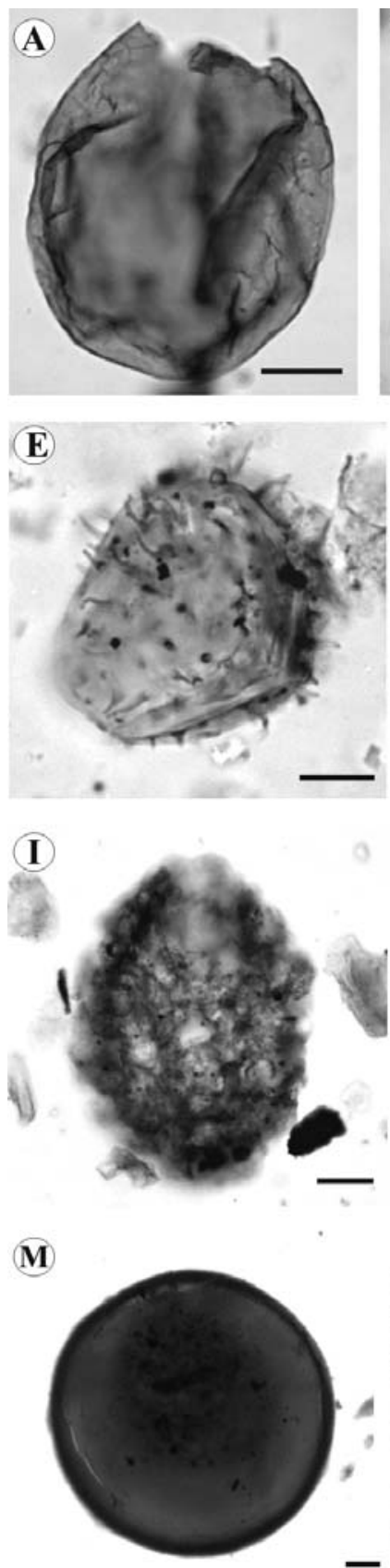
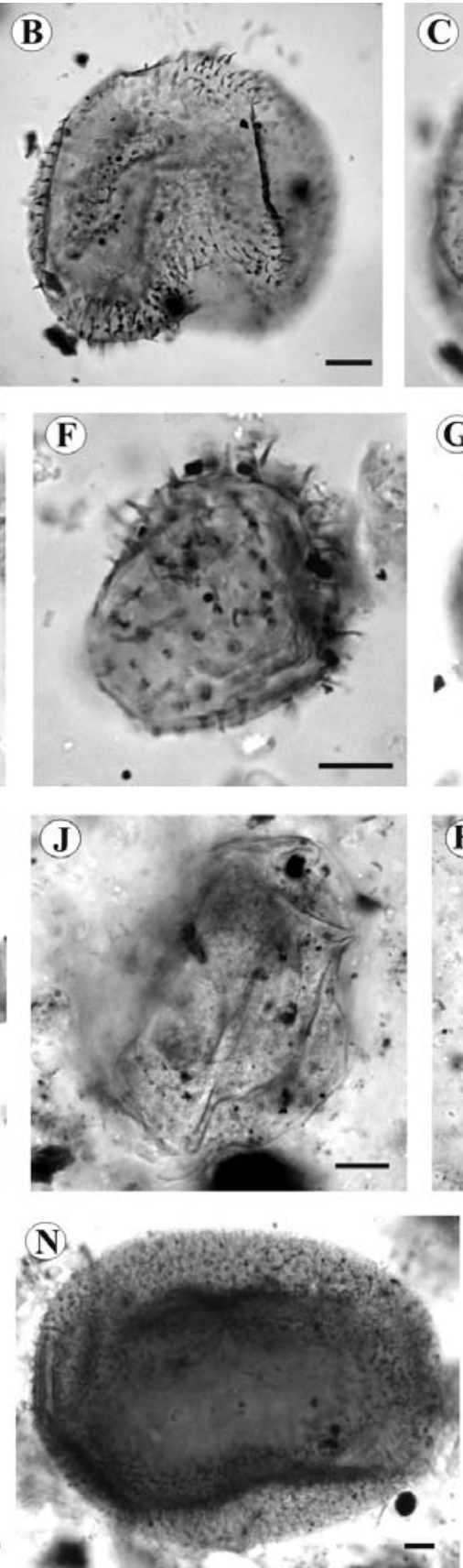
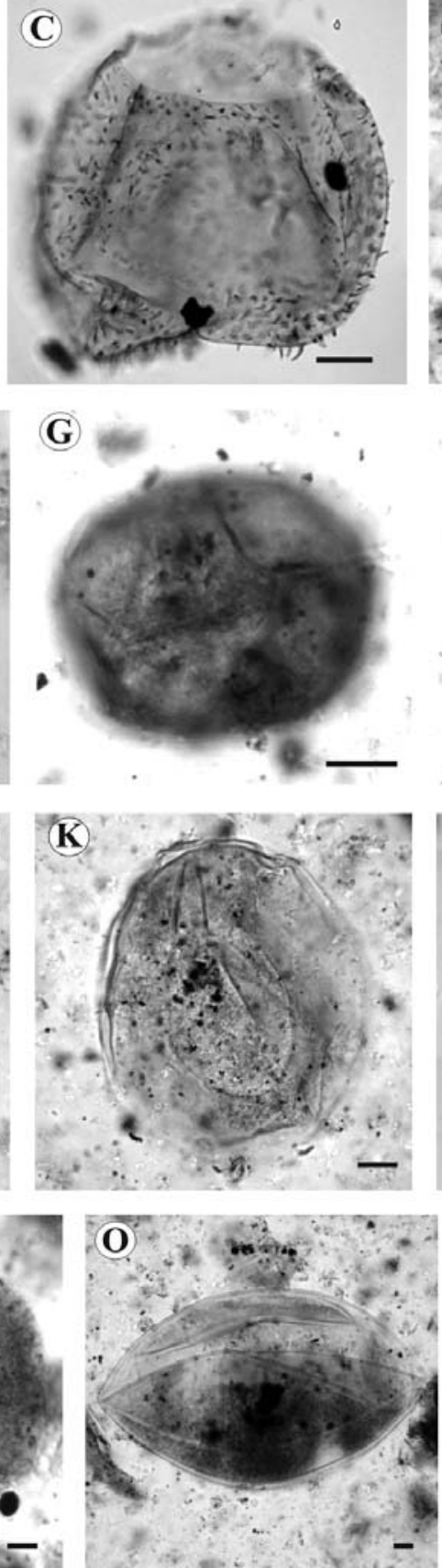
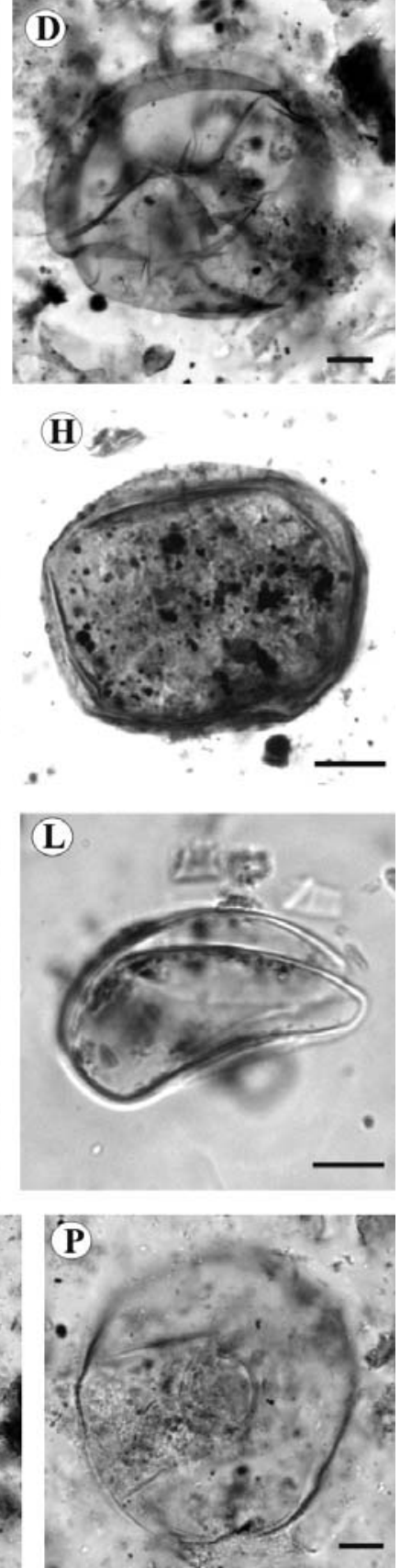

Figura 4. Palinomorfos acuáticos de la sección Albufera Lanushuaia. Número de muestra seguido por coordenadas England Finder. A, Brigantedinium spp., UNSP AL2637: E51/4. B-C, Votadinium spinosum Reid, UNSP AL2638: Q47/4; B, vista dorsal, foco bajo; C, vista dorsal, foco alto. D, Selenopemphix nephroides (Benedek) Benedek \& Sarjeant, UNSP AL2588: N18. E-F, Islandinium minutum (Harland \& Reid in Harland et al.) Head et al., UNSP AL2587: R44; E, foco alto; F, foco bajo. G-H, cf. Dubridinium sp., UNSP AL2579: U42/2; G, foco alto; H, foco bajo. I, Polykrikos schwartzii Bütschli, UNSP AL2638: Y27. J-K, Acritarco sp. 1; J, UNSP AL2584: L56/1; K, UNSP AL2584: T30/ 4. L, Spirogyra sp., UNSP AL1946: N54/1. M, Tasmanites sp., UNSP AL2581: H27/3. N-O, Huevo de copépodo; N, UNSP AL2588: P31/4; O, AL2586: B27/28. P, Halodinium sp., UNSP AL2580: A47. Escalas $=10 \mu \mathrm{m}$.

Figure 4. Aquatic palynomorphs from the Albufera Lanushuaia section. Number of sample followed by England Finder coordinates. A, Brigantedinium spp., UNSP AL2637: E51/4. B-C, Votadinium spinosum Reid, UNSP AL2638: Q47/4; B, dorsal view, low focus; C, dorsal view, high focus. D, Selenopemphix nephroides (Benedek) Benedek \& Sarjeant, UNSP AL2588: N18. E-F, Islandinium minutum (Harland \& Reid in Harland et al.) Head et al., UNSP AL2587: R44; E, high focus; F, low focus. G-H, cf. Dubridinium sp., UNSP AL2579: U42/2; G, high focus; H, low focus. I, Polykrikos schwartzii Bütschli, UNSP AL2638: Y27. J-K, Acritarco sp. 1; J, UNSP AL2584: L56/1; K, UNSP AL2584: T30/4. L, Spirogyra sp., UNSP AL1946: N54/1. M, Tasmanites sp., UNSP AL2581: H27/3. N-O, Copepod egg; N, UNSP AL2588: P31/4; O, AL2586: B27/28. P, Halodinium sp., UNSP AL2580: A47. Scale bars $=10 \mu \mathrm{m}$. 

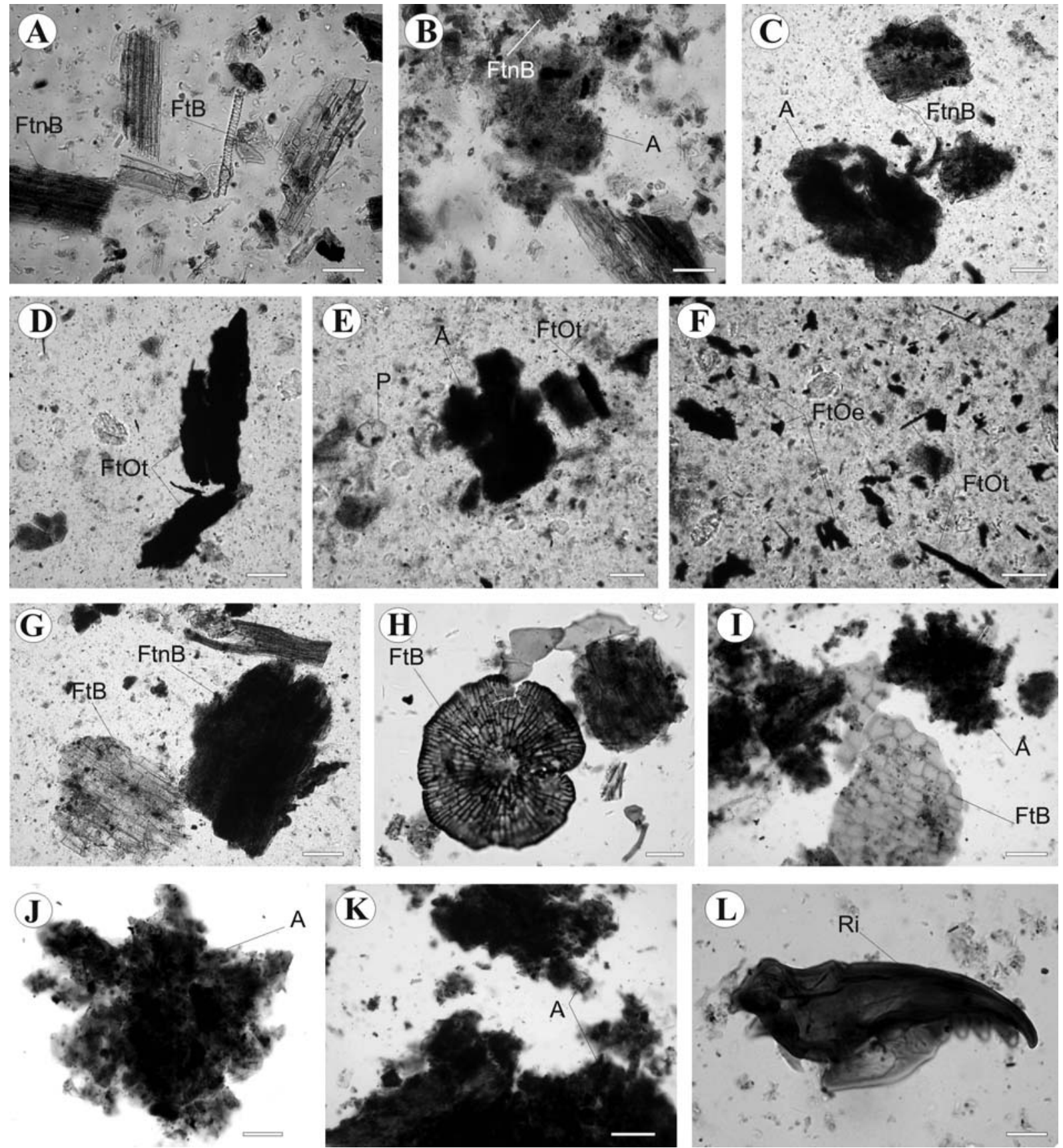

Figura 5. Palinofacies de la sección Albufera Lanushuaia. Número de muestra seguido por coordenadas de England Finder. A, palinofacies (x400) con detalle de fitoclastos translúcidos bioestructurados (FtB) y fitoclastos translúcidos no-bioestructurados (FtnB), UNSP AL2643: J30/4. B-C, palinofacies (x400) con detalle de fitoclastos translúcidos bioestructurados (FtB) y fitoclastos translúcidos no-bioestructurados (FtnB) junto con materia orgánica amorfa (A); B, UNSP AL2641: L42; C, UNSP AL2579: Q40/3. D-F, palinofacies (x400) con detalle de fitoclastos opacos en forma de tablillas (FtOt) y equidimensionales (FtOe), en algunos casos acompañados por palinomorfos y amorfo; $\mathbf{D}$, UNSP AL2579: T60/2; E, UNSP AL2582: T48/2; F, UNSP AL2583: E17/2. G-I, detalle (x400) de fitoclastos translúcidos no-bioestructurados (FtnB) junto con materia orgánica amorfa (A) y fitoclastos translúcidos bioestructurados (FtB): fruiting-body; G, UNSP AL2583: V29; H, UNSP AL1947: F49/4; I, UNSP AL1947: B48. J-K, detalle (x400) de amorfo granular (A); J, UNSP AL1946: E12/2; K, UNSP AL1946: F57/2. L, detalle $(x 400)$ de resto de invertebrado (Ri), UNSP AL1945: S29/4. Escalas $=30 \mu \mathrm{m}$.

Figure 5. Albufera Lanushuaia palynofacies (AL). Number of sample followed by England Finder coordinates. A, palynofacies ( $x 400)$ with detail of translucent biostructured phytoclasts (FTB) and translucent non-biostructured phytoclasts (FtnB), UNSP AL2643: J30/4. BC, palynofacies $(\mathrm{x} 400)$ with detail of translucent biostructured phytoclasts $(\mathrm{FtB})$ and translucent non-biostructured phytoclasts (FtnB) with amorphous organic matter (A); B, UNSP AL2641: L42; C, UNSP AL2579: Q40/3. D-F, palynofacies (x400) with detail of opaque phytoclasts in blade-shaped form (FtOt) and equidimensional (FtOe), in some cases accompanied by palynomorphs and amorphous; $\mathbf{D}$, UNSP AL2579: T60/2; E, UNSP AL2582: T48/2; F, UNSP AL2583: E17/2. G-I, detail (x400) of translucent non-biostructured phytoclasts (FtnB) with amorphous organic matter (A) and translucent biostructured phytoclasts (FtB): fruiting-body; G, UNSP AL2583: V29; H, UNSP AL1947: F49/4; I, UNSP AL1947: B48. J-K, detail (x400) of granular amorphous (A); J, UNSP AL1946: E12/2; K, UNSP AL1946: F57/2. L, detail (x400) of invertebrate remains (Ri),UNSP AL1945: S29/4. Scale bars $=30 \mu \mathrm{m}$. 


\section{DISCUSIÓN}

\section{Interpretación paleoambiental y paleoclimática}

Durante el Holoceno medio-tardío, la secuencia fósil de la Albufera Lanushuaia muestra altos valores de porcentaje y concentración de Nothofagus tipo dombeyi a través de la mayor parte del perfil sugiriendo la presencia del bosque cerrado de Nothofagus. La asociación de quistes de dinoflagelados está caracterizada por una baja diversidad de especies (nueve taxones identificados) así como también bajos valores de concentración. Los dinoquistes Peridiniales son dominantes, sugiriendo ambientes nerítico-internos (de Vernal \& Giroux, 1991). Los demás componentes de la materia orgánica palinológica están caracterizados por el predominio casi exclusivo de fitoclastos translúcidos. En las áreas de bosques, la abundancia de fitoclastos translúcidos podría estar en relación con una alta productividad de la cubierta vegetal local (Sebag et al., 2006).

Los cuatro tipos de materia orgánica palinológica identificados a lo largo de la sección estudiada no registran variaciones significativas en sus frecuencias relativas, por lo tanto no es posible determinar diferentes tipos de palinofacies en dicha secuencia. Todas las palinofacies analizadas muestran un dominio de fitoclastos translúcidos altamente degradados producto de la oxidación química por exposición aérea o subaérea de la materia orgánica asociados, en menor proporción, a materia orgánica amorfa y palinomorfos. Los fitoclastos opacos están escasamente representados. Esto indica la proximidad del área de aporte continental al ambiente marino, con distancias y/o tiempos de transporte relativamente cortos. Sebag et al. (2006) sugieren que las variaciones en la materia orgánica particulada estarían vinculadas a fluctuaciones del nivel del agua y/o a los influjos sedimentarios terrestres. La presencia de pirita indicaría condiciones reductoras, probablemente asociadas a ambientes marino-marginales.

Los resultados del análisis palinológico permiten observar a partir de los 5.800 años ${ }^{14} \mathrm{C}$ AP aproximadamente (Zona Palinológica AL-2) el desarrollo del bosque cerrado de Nothofagus y la presencia escasa de comunidades arbustivas y herbáceas (Poaceae, Empetrum, Gunnera, Asteraceae subf. Asteroideae y Asteraceae subf. Cichorioideae). Esta asociación vegetal sería análoga con la del Bosque Magallánico Deciduo que se desarrolla actualmente en el centro y sur de Tierra del Fuego con una precipitación media anual de 500-800 $\mathrm{mm}$ y temperaturas medias estivales entre 9 y $10^{\circ} \mathrm{C}$ (Pisano, 1977).

Entre los ca. 5.800 y $5.560 \pm 110$ años ${ }^{14} \mathrm{C}$ AP (Subzona Palinológica AL-2b) se registra una baja diversidad de especies de dinoquistes (cinco taxones identificados) y de concentraciones. La asociación de dinoquistes está representada por Brigantedinium spp., Echinidinium spp., Islandinium minutum, cf. Dubridinium sp., Selenopemphix nephroides, "round brown" y dinoquistes proximocorados indeterminados. Esta asociación sugiere un ambiente marinomarginal, con baja a moderada salinidad y altas concentraciones de nutrientes en las aguas superficiales, debido probablemente al aporte de agua dulce por derretimiento glaciar. La leve participación de una asociación de algas dulceacuícolas a marino salobre, confirma la descarga de agua dulce al ambiente marino. El registro de huevos de copépodos podría estar relacionado a los efectos combinados de alta productividad y altas tasas de sedimentación (van Waveren, 1994).

Con posterioridad a los $5.560 \pm 110$ años ${ }^{14} \mathrm{CAP}$ (Subzona Palinológica AL-2a), la asociación de dinoquistes está caracterizada por un leve incremento en la diversidad de especies (seis taxones identificados) y una disminución en la concentración de dinoquistes. La asociación está compuesta por Brigantedinium spp., Echinidinium spp., Selenopemphix quanta, cf. Selenopemphix quanta, Votadinium spinosum, Polykrikos kofoidii, P. schwartzii, "round brown" y dinoquistes proximocorados indeterminados. Esta asociación sugiere condiciones ambientales comparables con aquellas de la Subzona AL-2b.

El tramo superior de la secuencia (Zona Palinológica AL1, Post-bomb: 1958 DC), presenta una disminución en la frecuencia del polen arbóreo y el incremento polínico de arbustos y hierbas (Poaceae, Caryophyllaceae, Gunnera, Asteraceae subf. Asteroideae y Empetrum/Ericaceae) asociado a un incremento de huevos de copépodos y foraminíferos. Esto refleja el desarrollo de un bosque más abierto con áreas herbáceas adyacentes, en las proximidades de un ambiente marino.

\section{Comparación con otras localidades estudiadas}

De acuerdo con los datos paleoecológicos provenientes de turberas ubicadas en el sur de la Isla Grande de Tierra del Fuego (Heusser, 1998; Borromei \& Quattrocchio, 2008; Borromei et al., 2010), durante el Holoceno temprano se registra un ascenso de la temperatura en coincidencia con el inicio del Óptimo Termal Antártico (Bentley et al., 2009). Este mejoramiento climático es coincidente con un evento transgresivo a lo largo del Canal Beagle, el cual tuvo lugar alrededor de $c a .8 .000$ años ${ }^{14} \mathrm{C}$ AP. Hacia los $c a .7 .500$ años ${ }^{14} \mathrm{C} \mathrm{AP}$, de acuerdo con la asociación de moluscos, todo el área se habría transformado en un fiordo y las localidades de Río Ovando y Lago Roca en ambientes marino-someros (Gordillo et al., 2005).

En las áreas costeras, los registros polínicos revelan con anterioridad al evento transgresivo, el desarrollo de paleocomunidades vegetales propias del ecotono bosqueestepa en concordancia con el patrón de vegetación regional (Heusser, 2003; Borromei \& Quattrocchio, 2008). Esto habría sido favorecido por el incremento de la temperatura y niveles de humedad efectiva más bajos que los actuales, permitiendo la expansión del bosque y la estepa a lo largo del Canal Beagle (Heusser, 1998). Estas condiciones ambientales también se registraron en el suroeste de la Patagonia $\left(50^{\circ} \mathrm{S}\right)$ (Mancini, 2009), en el Estrecho de Magallanes (53 ${ }^{\circ}$ ) (McCulloch \& Davies, 2001) y en el sector oeste de Isla de los Estados, unos $130 \mathrm{~km}$ al este del Canal Beagle (Ponce, 2009). La presencia del bosque y un clima más árido habrían favorecido un incremento en los incendios de carácter regional en el 
centro-sur de Tierra del Fuego y sur de Patagonia (Huber et al., 2004; Whitlock et al., 2007). Estas condiciones se habrían extendido hasta los aproximadamente 6.000-5.500 años ${ }^{14} \mathrm{C}$ AP, favorecidas por la migración de los vientos del Oeste hacia latitudes más altas, reducción de la capa de hielo antártico y disminución del gradiente térmico entre el ecuador y los polos (Whitlock et al., 2007).

Durante la incursión marina holocena, la vegetación litoral en el área del Canal Beagle fue principalmente arbórea registrándose un aumento en las frecuencias del polen de Nothofagus, sugiriendo mayor disponibilidad hídrica por la acción moderadora del mar, mientras el ecotono bosqueestepa se extendía regionalmente hacia el interior de la isla (Grill et al., 2002; Borromei \& Quattrocchio, 2007, 2008).

Las asociaciones palinológicas identificadas en las localidades de Bahía Lapataia (5450'S-68³4'O; oeste del Canal Beagle) (Borromei \& Quattrocchio, 2007) y Río Varela (54 $52^{\prime}$ 'S-67 $7^{\circ} 11^{\prime} \mathrm{O}$; este del Canal Beagle) (Grill et al., 2002) (Figura 1) revelan entre los ca. 8.550 y 5.800 años ${ }^{14} \mathrm{C} \mathrm{AP} \mathrm{y} \mathrm{a}$ los 6.240 años ${ }^{14} \mathrm{C}$ AP y 6.060 años ${ }^{14} \mathrm{C}$ AP, respectivamente, ambientes marino-marginales con predominio de palinomorfos terrestres sobre marinos, indicando un gran aporte fluvial. Los palinomorfos marinos están caracterizados por una escasa asociación de quistes de dinoflagelados principalmente dominada por taxones Peridiniales (Brigantedinium spp., Selenopemphix sp.) además de taxones Gonyaulacales (Spiniferites spp., Operculodinium centrocarpum Wall \& Dale). Los acritarcos como Halodinium sp. y zoomorfos, principalmente foraminíferos y huevos de copépodos, son abundantes. La asociación de palinomorfos marinos refleja ambientes estuarinos internos a externos relacionados con salinidades bajas y variables, y/o turbulencia, temperatura del agua de mar templado-fría y abundancia de nutrientes disueltos debido al aporte de agua dulce por escurrimiento superficial (Borromei \& Quattrocchio, 2007; Grill et al., 2002).

Durante el Holoceno medio los espectros polínicos reflejan el desarrollo de un bosque cerrado de Nothofagus a partir de los 6.000-5.500 años ${ }^{14} \mathrm{CAP}$ aproximadamente, bajo condiciones más frías y húmedas que en el Holoceno temprano, extendiéndose no solamente en las áreas bajas costeras a lo largo del Canal Beagle, sino también en los valles interiores y valles de altura de los Andes Fueguinos (Heusser, 2003; Borromei et al., 2007, 2010). Las condiciones de mayor humedad efectiva y descenso de temperatura, que habrían favorecido el desarrollo y expansión del bosque de Nothofagus y la abrupta disminución de los incendios regionales, se relacionan con la intensificación y/o migración latitudinal de los vientos del Oeste entre los $50-54^{\circ} \mathrm{S}$, afectando el sur de Patagonia y Andes Fueguinos (Villa Martínez \& Moreno, 2007; Waldmann et al., en prensa; Mancini, 2009; Borromei et al., 2010).

De acuerdo con los datos palinológicos provenientes de los sedimentos marinos, las paleocomunidades vegetales reflejan para las áreas costeras el mismo patrón de vegetación regional que se observa en el centro-sur de la Isla Grande de Tierra del Fuego, con desarrollo del bosque cerrado de
Nothofagus y escasa presencia de comunidades arbustivas y herbáceas.

En el área del Archipiélago Cormoranes (O del Canal Beagle), el análisis palinológico de la sección Río Ovando (5451'S-68 35'O) (Figura 1) muestra porcentajes significativos de Nothofagus registrados a lo largo de la mayor parte del perfil, sugiriendo la presencia del bosque cerrado de Nothofagus bajo condiciones frías y de mayor humedad efectiva (Candel et al., 2009). El intervalo comprendido entre los 4.160 y 3.542 años ${ }^{14} \mathrm{C}$ AP, registra una mayor diversidad de especies (nueve taxones identificados) y bajas concentraciones de dinoquistes.

La asociación de dinoquistes identificada (Islandinium minutum, cf. Islandinium minutum, Echinidinium spp., acompañados por Brigantedinium simplex, B. spp., Selenopemphix quanta, S. spp., cf. Pentapharsodinium dalei, Polykrikos kofoidii, P. schwartzii, Operculodinium cf. centrocarpum y Spiniferites spp.) sugiere un ambiente marino-marginal, con baja a moderada salinidad y altas concentraciones de nutrientes en las aguas marinas superficiales, debido probablemente al aporte de agua dulce por escurrimiento superficial. Estas asociaciones indican condiciones similares a aquellas registradas en la Albufera Lanushuaia a partir de los 5.750 años ${ }^{14} \mathrm{CAP}$ (Zona Palinológica AL-2).

En la sección de Río Ovando, este intervalo caracterizado por una asociación con relativamente alta diversidad de especies y bajas concentraciones de dinoquistes, es interrumpido entre los 4.160 y 4.064 años ${ }^{14} \mathrm{C}$ AP por una asociación de dinoquistes dominada exclusivamente por el complejo Islandinium-Echinidinium, presentando la mayor abundancia y menor diversidad de especies de dinoquistes de la secuencia. Dicha asociación podría indicar la ocurrencia de "especies oportunistas" sugiriendo un alto aporte de agua dulce por escorrentías superficiales (Candel et al., 2009). La baja diversidad de las asociaciones de microplancton podría ser indicativa de condiciones restringidas y estrés ambiental con salinidades, a menudo, inestables (Gorin \& Steffen, 1991).

Con posterioridad a los 3.000 años, en la sección de Río Ovando se observa una disminución en el registro del polen de Nothofagus y un aumento en el polen de hierbas y arbustos con un incremento de algas Prasinophyceae (Cymatiosphaera sp.) y Zygnemataceae (Spirogyra sp.). Esta asociación de palinomorfos, sugiere el desarrollo de un bosque abierto de Nothofagus con áreas de pastizales y arbustos, con descarga fluvial al ambiente marino indicado por la ocurrencia de palinomorfos acuáticos dulceacuícolas a salobres, relacionados con un evento regresivo (Candel et al., 2009; Candel, 2010). Isla (1989) reconoció que el episodio de máximo transgresivo en Tierra del Fuego ocurrió entre los 9.000 y 7.000 años ${ }^{14} \mathrm{C}$ AP. Aquellos depósitos más recientes representarían fases regresivas de la línea de costa.

En el tramo superior de la sección Albufera Lanushuaia (Zona Palinológica AL-1, Post-bomb: 1.958 DC), la asociación de palinomorfos es similar a la descripta con posterioridad a los 3.000 años en Río Ovando. Este patrón de vegetación también es registrado en los tramos superiores de las 
secuencias de Bahía Lapataia y Río Varela para las áreas costeras, con la presencia del bosque de Nothofagus asociado a un mayor desarrollo de áreas herbáceo-arbustivas y escasa ocurrencia de palinomorfos acuáticos, sugiriendo un nivel del mar bajo durante el Holoceno tardío (Grill et al., 2002; Borromei \& Quattrocchio, 2007).

\section{CONCLUSIONES}

El análisis de los constituyentes de la materia orgánica palinológica (palinofacies) en la sección de Albufera Lanushuaia muestra una dominancia de fitoclastos translúcidos acompañados en forma subordinada por materia orgánica amorfa y en menor proporción por palinomorfos. Los fitoclastos opacos están escasamente representados. Esto sugiere una cercanía al área de aporte continental con tiempos y/o distancias de transporte relativamente cortos. El predomino de fitoclastos translúcidos asociados a pirita sugiere condiciones disóxicas del medio de depositación.

La vegetación durante el Holoceno medio-tardío en las áreas costeras estaría caracterizada por el dominio del bosque de Nothofagus y escasa presencia de comunidades arbustivas y herbáceas bajo condiciones frías y de mayor humedad efectiva.

La asociación de dinoquistes identificada en la localidad de la Albufera Lanushuaia se encuentra dominada por taxones Peridiniales como Brigantedinium spp., Islandinium minutum, Echinidinium granulatum, E. delicatum, E. spp., Selenopemphix quanta, Selenopemphix nephroides, Votadinium spinosum, Pentapharsodinium dalei, cf. Dubridinium sp., acompañados por Polykrikos kofoidii, $P$. schwartzii, que sugiere ambientes marino-marginales, con baja a moderada salinidad y altas concentraciones de nutrientes, debido probablemente al aporte de agua dulce por escorrentía superficial.

Por comparación, los sedimentos marinos del Holoceno medio-tardío (Albufera Lanushuaia-Río Ovando) registran asociaciones con mayor diversidad de especies de dinoquistes que aquellas identificadas en los depósitos marinos del Holoceno temprano-medio (Bahía Lapataia y Río Varela). La diversificación de especies sugiere un incremento en la paleoproductividad marina debido al aporte de nutrientes por escorrentía superficial en relación con un aumento en las precipitaciones. Evidencias paleoecológicas provenientes del sur de Patagonia y Tierra del Fuego señalan un aumento en las precipitaciones a partir de los $c a$. 6.000 años AP, en relación con una intensificación y/o migración de los vientos del Oeste.

Durante los últimos 3.000 años AP, en las áreas costeras a lo largo del Canal Beagle, las asociaciones de palinomorfos sugieren el desarrollo de un bosque abierto de Nothofagus con áreas de pastizales y arbustos y escasa ocurrencia de palinomorfos acuáticos (algas Prasinophyceae y zoomorfos), relacionados con un evento regresivo.

\section{AGRADECIMIENTOS}

Los autores desean agradecer a A. Coronato, demás integrantes del Laboratorio de Geología del Cuaternario
(CADIC-CONICET), D. Mauquoy y M. Blaauw por la ayuda brindada durante las tareas de campo. A P.A. de Souza por su cordial ayuda en la revisión del abstract en portugués. A la Agencia de Promoción Científica y Tecnológica - FONCyT (PICT Redes 2002 Nro. 00067), por el financiamiento económico de este estudio. Los aportes realizados por K.B. Meyer, un revisor anónimo y editores contribuyeron a mejorar este trabajo.

\section{REFERENCIAS}

Albero, M.C.; Angiolini, F. \& Piana, L. 1988. Holocene ${ }^{14}$ C reservoir effect at Beagle Channel (Tierra del Fuego, Argentina Republic). Quaternary of South America and Antarctic Peninsula, 5:59-71.

Bentley, M.J.; Hodgson; D.A.; Smith, J.A.; Cofaigh, C.O.; Domack, E.W.; Larter, R.D.; Roberts, S.J.; Brachfeld, S.; Leventer, A.; Hjort, C.; Hillenbrand, C-D. \& Evans, J. 2009. Mechanisms of Holocene palaeoenvironmental change in the Antarctic Peninsula region. The Holocene, 19:51-69. doi:10.1177/0959683608096603

Birks, H.J. \& Birks, H.H. 1980. Quaternary palaeoecology. Londres, Arnold Publishers, 289 p.

Birks, H.J. \& Gordon, A.D. 1985. Numerical methods in Quaternary pollen analysis. Londres, Academic Press, 313 p.

Borromei, A.M.; Coronato, A.; Franzén, L.; Ponce, J.P.; López Sáez, J.A.; Maidana, N.; Rabassa, J. \& Candel, M.S. 2010. Multiproxy record of Holocene paleoenvironmental change, Tierra del Fuego, Argentina. Palaeogeography, Palaeoclimatology, Palaeoecology, 286:1-16. doi:10.1016/j.palaeo.2009.11.033

Borromei, A.M.; Coronato, A.; Quattrocchio, M.; Rabassa, J.; Grill, S. \& Roig, C. 2007. Late Pleistocene-Holocene environments in Valle Carbajal, Fuegian Andes valley, southern South America. Journal of South American Earth Sciences, 23:321-335. doi:10.1016/j.jsames.2007.02.008

Borromei, A.M. \& Quattrocchio, M. 2001. Palynological study of Holocene marine sediments from Bahía Lapataia, Beagle Channel, Tierra del Fuego, Argentina. Revista Española de Micropaleontología, 33:61-70.

Borromei, A.M. \& Quattrocchio, M. 2007. Holocene sea-level change and marine palynology of the Beagle Channel, southern Tierra del Fuego, Argentina. Ameghiniana, 44:161-171.

Borromei, A.M. \& Quattrocchio, M. 2008. Late and postglacial paleoenvironments of Tierra del Fuego: terrestrial and marine palynological evidence. In: J. Rabassa (ed.) The Late Cenozoic of Patagonia and Tierra del Fuego, Elsevier, p. 369-381.

Borromei,A.; Quattrocchio, M. \& Rabassa, J. 1997. Estudio palinológico de sedimentos marinos holocénicos en Bahía Lapataia, Tierra del Fuego, Argentina. In: CONGRESSO DA ASSOCIAÇÃO BRASILEIRA PARA ESTUDOS DO QUATERNARIO, 6, 1997. Abstracts, Curitiba, ABEQUA, p. 317-321.

Bujalesky, G.; Coronato, A.; Roig, C. \& Rabassa, J. 2004. Holocene differential tectonic movements along the argentine sector of the Beagle Channel (Tierra del Fuego) inferred from marine palaeoenvironments. Bollettino di Geofisica Teorica e Applicata, 45:235-238.

Candel, M.S. 2010. Cambios relativos del nivel del mar en el Canal Beagle, Tierra del Fuego (Cenozoico Tardí), en base al análisis palinológico. Universidad Nacional del Sur, Tesis Doctoral, $194 \mathrm{p}$.

Candel, M.S.; Borromei, A.M.; Martínez, M.A.; Gordillo, S.; Quattrocchio, M. \& Rabassa, J. 2009. Middle-Late Holocene palynology and marine mollusks from Archipiélago Cormoranes area, Beagle Channel, southern Tierra del Fuego, Argentina. 
Palaeogeography, Palaeoclimatology, Palaeoecology, 273:111122. doi:10.1016/j.palaeo.2008.12.009

Codignotto, J.O. 1984. Estratigrafía y geomorfología del PleistocenoHoloceno costanero entre los paralelos 5330' Sur y $42^{\circ} 00^{\prime}$ Sur, Argentina. In: CONGRESO GEOLÓGICO ARGENTINO, 9, 1984. Actas, San Carlos de Bariloche, v. 3, p. 513-519.

Dale, B. 1976. Cyst formation, sedimentation, and preservation: factors affecting dinoflagellate assemblages in Recent sediments from Trondheimsfjord, Norway. Review of Palaeobotany and Palynology, 22:39-60. doi:10.1016/0034-6667(76)90010-5

Delcourt, P.A. \& Delcourt, H.R. 1980. Pollen preservation and Quaternary environmental history in the southeastern United Stated. Palynology, 4:215-231.

de Vernal, A. \& Giroux, L. 1991. Distribution of organic walled microfossils in Recent sediments from the Estuary and Gulf of St. Lawrence: some aspects of the organic matter fluxes. Canadian Journal of Fisheries and Aquatic Science, 113:189-199.

Fensome, R.A. \& Williams, G.L. 2004. The Lentin and Williams index of fossil dinoflagellates 2004 edition. Pittsburgh, American Association of Stratigraph Palynologists, 909 p. (Contributions Series 42).

Gordillo, S. 1993. Las terrazas marinas holocenas de la región del Beagle (Tierra del Fuego) y su fauna asociada. In: CONGRESO GEOLÓGICO ARGENTINO, 12, 1993. Actas, Mendoza, v. 6, p. 34-39.

Gordillo, S.; Bujalesky, G.; Pirazzoli, P.; Rabassa, J. \& Saliége, J. 1992. Holocene raised beaches along the northern coast of the Beagle Channel, Tierra del Fuego, Argentina. Palaeogeography, Palaeoclimatology, Palaeoecology, 99:41-54. doi:10.1016/00310182(92)90006- $Q$

Gordillo, S.; Coronato, A. \& Rabassa, J. 1993. Late Quaternary evolution of a subantarctic paleofjord, Tierra del Fuego. Quaternary Science Reviews, 12:889-897. doi:10.1016/0277-3791(93)90027-J

Gordillo, S.; Coronato, A. \& Rabassa, J. 2005. Quaternary molluscan faunas from the island of Tierra del Fuego after the Last Glacial Maximum. Scientia Marina, 69(Supl. 2):337-348.

Gorin, G.E. \& Steffen D. 1991. Organic facies as a tool for recording eustatic variations in marine fine-grained carbonates - example of the Berriasian stratotype at Berrias (Ardèche, SE France). Palaeogeography, Palaeoclimatology, Palaeoecology, 85:303320. doi:10.1016/0031-0182(91)90164-M

Grill, S.; Borromei, A.M.; Quattrocchio, M.; Coronato, A.; Bujalesky, G. \& Rabassa, J. 2002. Palynological and sedimentological analysis of Recent sediments from Río Varela, Beagle Channel, Tierra del Fuego, Argentina. Revista Española de Micropaleontología, 34:145-161.

Grimm, E. 2004. TGView 2.0.2 Springfield, Illinois State Museum/ Research and Collection Center.

Head, M.; Harland, R. \& Matthiessen, J. 2001. Cold marine indicators of the late Quaternary: the new dinoflagellate cyst genus Islandinium and related morphotypes. Journal of Quaternary Science, 16:621-636. doi:10.1002/jqs.657

Heusser, C.J. 1998. Deglacial paleoclimate of the American sector of the Southern Ocean: Late Glacial-Holocene records from the latitude of Canal Beagle $\left(55^{\circ} \mathrm{S}\right)$, Argentine Tierra del Fuego. Palaeogeography, Palaeoclimatology, Palaeoecology, 141:277301. doi:10.1016/S0031-0182(98)00053-4

Heusser, C.J. 2003. Ice age southern Andes - A chronicle of paleoecological events. Amsterdam, Elsevier, $240 \mathrm{p}$.

Heusser, L. \& Stock, C. 1984. Preparation techniques for concentrating pollen from marine sediments and other sediments with low pollen density. Palynology, 8:225-227.
Huber, U.M.; Markgraf, V. \& Schäbitz, F. 2004. Geographical and temporal trends in Late Quaternary fire histories of FuegoPatagonia, South America. Quaternary Science Reviews, 23:1079-1097. doi:10.1016/j.quascirev.2003.11.002

Isla, F.I. 1989. Holocene sea-level fluctuations in the Southern Hemisphere. Quaternary Science Reviews, 8:359-368. doi:10.1016/0277-3791(89)90036-X

Isla, F.; Bujalesky, G. \& Coronato, A. 1999. Procesos estuarinos en el canal Beagle, Tierra del Fuego. Revista de la Asociación Geológica Argentina, 54:307-318.

Iturraspe, R.; Sottini, R.; Schroeder, C. \& Escobar, J. 1989. Hidrologia y variables climaticas del Territorio de Tierra del Fuego. Ushuaia, Centro Austral de Investigaciones Cientificas, 196 p. (Contribución Científica 7).

Mancini, M.V. 2009. Holocene vegetation and climate changes from a peat pollen record of the forest-steppe ecotone, Southwest of Patagonia (Argentina). Quaternary Science Reviews, 28:14901497. doi:10.1016/j.quascirev.2009.01.017

McCormac, F.G.; Hogg, A.G.; Blackwell, P.G.; Buck, C.E.; Higham, T.F.G. \& Reimer, P.J. 2004. SHCal04 Southern Hemisphere Calibration 0-11.0 cal kyr BP. Radiocarbon, 46:1087-1092.

McCulloch, R.D. \& Davies S. J. 2001. Late glacial and Holocene palaeoenvironmental changes in the central Strait of Magellan, southern Patagonia. Palaeogeography, Palaeoclimatology, Palaeoecology, 173:143-173. doi:10.1016/S0031-0182(01)00316-9

Overpeck, J.T.; Webb, T. \& Prentice, I.C. 1985. Quantitative interpretation of fossil pollen spectra: dissimilary coefficients and methods of modern analogs. Quaternary Research, 23:87108. doi:10.1016/0033-5894(85)90074-2

Pals, J.P.; van Geel, B. \& Delfos, A. 1980. Paleoecological studies in the Klokkeweel bog near Hoogkarspel (Prov. of NoordHolland). Review of Palaeobotany and Palynology, 30:371-418. doi:10.1016/0034-6667(80)90020-2

Pisano, E. 1977. Fitogeografía de Fuego-Patagonia chilena. Comunidades vegetales entre las latitudes $52^{\circ}$ y $56^{\circ} \mathrm{S}$. Anales del Instituto de la Patagonia, 8:121-250.

Ponce, J.F. 2009. Palinología y geomorfología del Cenozoico tardio de la Isla de los Estados. Universidad Nacional del Sur, Tesis Doctoral, $191 \mathrm{p}$.

Porter, S.C.; Stuiver, M. \& Heusser, C.J. 1984. Holocene sea-level changes along the Strait of Magellan and Channel, southernmost South America. Quaternary Research, 22:59-67. doi:10.1016/ 0033-5894(84)90006-1

Prentice, I.C. 1986. Multivariate methods of data analysis. In: B.E. Berglund (ed.) Handbook of Holocene Palaeocology and Palaeohydrology, John Wiley and Sons, p. 799-816.

Rabassa, J.; Bujalesky, G.; Meglioli, A.; Coronato, A.; Gordillo, S.; Roig, C. \& Salemme, M. 1992. The Quaternary of Tierra del Fuego, Argentina: the status of our knowledge. Sveriges Geologiska Undersköning, 81:249-256.

Rabassa, J.; Coronato,A.; Bujalesky, G.; Salemme, M.; Roig, C.; Meglioli, A.; Heusser, J.; Gordillo, S.; Roig, F.; Borromei, A. \& Quattrocchio, M. 2000. Quaternary of Tierra del Fuego, Southernmost South America: an updated review. Quaternary International, 68-71:217240. doi:10.1016/S1040-6182(00)00046-X

Rabassa, J.; Coronato, A.; Gordillo, S.; Candel, M.S. \& Martínez, M.A. 2009. Paleoambientes litorales durante el inicio de la transgresión marina holocena en Bahía Lapataia, Canal Beagle, Parque Nacional Tierra del Fuego. Revista de la Asociación Geológica Argentina, 65:648-659.

Rabassa, J.O.; Heusser, C.J. \& Stuckenrath, R. 1986. New data on Holocene sea transgression in the Beagle Channel: Tierra del 
Fuego, Argentina. Quaternary of South America and Antarctic Peninsula, 4:291-309.

Reimer, P.J.; Brown, T.A. \& Reimer, R.W. 2004. Discussion: reporting and calibration of post-bomb ${ }^{14} \mathrm{C}$ data. Radiocarbon, 46:1299-1304.

Rochon, A.; de Vernal, A.; Turon, J.L.; Matthiessen, J. \& Head, M.J. 1999. Distribution of dinoflagellate cysts in surface sediments from the North Atlantic Ocean and adjacent seas in relation to seasurface parameters. Pittsburgh, American Association of Stratigraph Palynologists, 152 p. (Contribution Series 35).

Rutter, N.W.; Schnack, E.J.; Fasano, J.L.; Isla, F.I.; del Río, J. \& Radtke, U. 1989. Correlation and dating of Quaternary littoral zones along the Patagonian coast, Argentina. Quaternary Science Reviews, 8:213-234. doi:10.1016/0277-3791(89)90038-3

Sebag, D.; Copard, Y.; Di-Giovanni, Ch.; Durand, A.; Laignel, B.; Ogier, S. \& Lallier-Verges, E. 2006. Palynofacies as useful tool to study origins and transfers of particulate organic matter in recent terrestrial environments: synopsis and prospects. Earth-Science Reviews, 79:241-259. doi:10.1016/j.earscirev.2006.07.005

Stockmarr, J. 1971. Tablets with spores used in absolute pollen analysis. Pollen et Spores, 13:615-621.

Stuiver, M. \& Reimer, P. 1993. Extended ${ }^{14} \mathrm{C}$ database and revised CALIB radiocarbon calibration program. Radiocarbon, 35:215-230.

Stuiver, M.; Reimer, P.J. \& Reimer, R.W. 2005. Calib 5.0.2. Available at http://calib.qub.ac.uk/calib/; accessed on 10/03/2010.

Tyson, R. 1995. Sedimentary organic matter. Londres, Chapman \& Hall, $615 \mathrm{p}$. van Geel, B.; Hallewas, D.P. \& Pals, J.P. 1983. A late Holocene deposit under the Westfriese Zeedijk near Enkhuizen (Prov. of Nood-Holland, The Netherlands): palaeoecological and archaeological aspects. Review of Palaeobotany and Palynology, 38:269-335. doi:10.1016/0034-6667(83)90026-X

van Waveren, I. M. 1994. Distribution of copepod egg-envelopes in sub- Recent sediments from the Banda Sea (Indonesia). Scripta Geologica, 105:53-67.

Villa-Martínez, R. \& Moreno, P.I. 2007. Pollen evidence for variations in the southern margin of the westerly winds in SW Patagonia over the last 12,600 years. Quaternary Research, 68:400-409. doi:10.1016/j.yqres.2007.07.003

Waldmann, N.; Ariztegui, D.; Anselmetti, F.S.; Austin Jr., J.A.; Moy, C.M.; Stern, C.; Recasens, C. \& Dunbar, R.B. (en prensa). Holocene climatic fluctuations and positioning of the Southern Hemisphere Westerlies in Tierra del Fuego $\left(54^{\circ} \mathrm{S}\right)$, Patagonia. Journal of Quaternary Science. doi:10.1002/jqs. 1263

Whitlock, C.; Moreno, P. \& Bartlein, P. 2007. Climatic controls of Holocene fire patterns in southern South America. Quaternary Research, 68:28-36. doi:10.1016/j.yqres.2007.01.012

Zonneveld, K.A.F. 1997. New species of organic walled dinoflagellate cysts from modern sediments of the Arabian Sea (Indian Ocean). Review of Palaeobotany and Palynology, 97:319-337. doi:10.1016/S0034-6667(97)00002-X

Received in May, 2011; accepted in November, 2011.

Apéndice 1. Descripciones de microplancton de pared orgánica con asignación abierta.

Appendix 1. Descriptions of organic-walled microplankton remained in open nomenclature.

División DINOFLAGELLATA (Bütschli, 1885) Fensome et al., 1993

Clase DINOPHYCEAE Pascher, 1914

Subclase PERIDINIPHYCIDAE Fensome et al., 1993

Orden PERIDINIALES Haeckel, 1894

Suborden PERIDININEAE (autonimia)

Familia PROTOPERIDINIACEAE Balech, 1988 nom. cons.

Subfamilia PROTOPERIDINIOIDAE Balech, 1988

Género Selenopemphix Benedek, 1972 emend. Head, 1993

Selenopemphix quanta (Bradford, 1975) Matsuoka, 1985

cf. Selenopemphix quanta

Material estudiado. UNSP 2637: O49/4.

Descripción. Quiste con compresión polar de forma sub-circular, cuya pared es lisa de color marrón. Procesos sólidos, acuminados y truncados, con bases levemente expandidas y homogeneamente distribuidos. El arqueopilo no fue observado en los ejemplares estudiados. Dimensiones. Diámetro (ancho) del cuerpo central: 30-(38,4)-48,8 $\mu \mathrm{m}$. Longitud de los procesos: 5-(9,8)-13 um (1 ejemplar medido). Observaciones. Estos ejemplares se comparan y no se asignan a Selenopemphix quanta por registrar un diámetro menor del cuerpo central (S. quanta: 40-83 um in Rochon et al., 1999), baja densidad y menor longitud de los procesos (S. quanta: 10-16 $\mu \mathrm{m}$ in Rochon et al., 1999) sin bases expandidas.

Subfamilia DIPLOPSALIOIDAE Abé, 1981

Género Dubridinium Reid, 1977

cf. Dubridinium sp.

(Figuras 4G-H)

Material estudiado. UNSP 2582: K50/4; UNSP 2579: U42/2.

Descripción. Quiste proximado de contorno sub-circular y color marrón oscuro. Pared compuesta por dos capas, endofragma granulado y perifragma liso. Arqueopilo no discernible. Se observa la cicatriz flagelar.

Dimensiones. Diámetro máximo del cuerpo: 46,4-(53)-64 $\mu \mathrm{m}$ (2 ejemplares medidos). 
Grupo ACRITARCHA Evitt, 1963

Género Halodinium Bujak, 1984

\author{
Halodinium sp. \\ (Figura 4P)
}

Material estudiado. UNSP 2588: P16/2; UNSP 2586: H36; UNSP 2581: K56/2; UNSP 2580: A47, D14/2.

Descripción. Palinomorfo discoidal aplanado. La pared del cuerpo es lisa a microgranulada, delgada y hialina. La membrana externa se extiende alrededor del cuerpo central, es delgada y a menudo se presenta incompleta por rotura. El piloma central es circular con un borde liso bien definido, usualmente engrosado.

Dimensiones. Diámetro del cuerpo central (excluyendo la membrana): 28,8-(60)-92 $\mu \mathrm{m}$. Diámetro del piloma: 9,6-(17)39,9 $\mu \mathrm{m}$ (5 ejemplares medidos).

Observaciones. Algunos ejemplares presentan un cuerpo central más pequeño con delgadas costillas radiales.

\title{
Acritarco sp. 1
}

(Figuras 4J-K)

Material estudiado. UNSP 2584: V53/3, L56/1, T30/4.

Descripción. Palinomorfo de contorno sub-circular, pared gruesa, psilada a microgranulada y pliegues concéntricos. Presenta un piloma circular de contorno bien definido; en algunos casos se observa una membrana muy delgada rodeando el cuerpo.

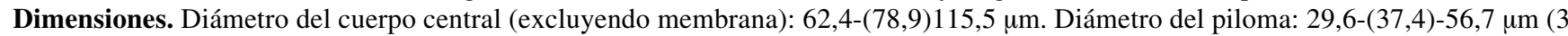
ejemplares medidos).

Observaciones. Usualmente se observa el opérculo del piloma en el interior del cuerpo.

División CHAROPHYTA sensu Lewis \& McCourt, 2004

Clase ZYGNEMATOPHYCEAE van den Hoek et al., 1995

Orden ZYGNEMATALES G.M. Smith, 1933

Familia ZYGNEMATACEAE Kützing, 1843

Género Spirogyra Link, 1820

\section{Spirogyra sp \\ (Figura 4L)}

Material estudiado. UNSP 2579: T40/1; UNSP 1946: N54/1, C48/3; UNSP 1945:Y46, Z27/3.

Descripción. Espora de contorno elíptico (extremos agudos a subredondeados) y pared delgada. La superficie de la pared es lisa, en algunos casos, escabrada. Sutura longitudinal que no rodea completamente la espora permitiendo que las valvas permanezcan unidas al abrirse.

Dimensiones. Diámetro máximo 24-(35,6)-42 $\mu \mathrm{m}$ (5 ejemplares medidos).

División PRASINOPHYTA Round, 1971

Clase PRASINOPHYCEAE Parke (in Parke \& Dixon, 1964)

Orden PTEROSPERMATALES Parke \& Green (in Parke \& Dixon, 1976)

Familia TASMANACEAE Sommer, 1956 emend. Mädler, 1963

Género Tasmanites Newton, 1875

\section{Tasmanites sp.}

(Figura 4M)

Material estudiado. UNSP 2581: H27/3.

Descripción. Cuerpo de contorno circular y pared gruesa. La superficie de la pared está ornamentada con gránulos subcirculares hasta poligonales de bajo relieve, distribuidos uniformemente dando el aspecto de un panal de abejas. El centro de cada gránulo es atravesado por un diminuto canal, que en algunos casos no penetra toda la pared. Se observa una hendidura en forma de "v", posiblemente para la liberación del contenido celular.

Dimensiones. Diámetro del cuerpo: 130,2-(131,2)-132,3 $\mu \mathrm{m}$. Espesor de la pared: 5-(5,6)-6,3 $\mu \mathrm{m}$ (1 ejemplar medido). 